\title{
Produção de enterotoxinas por Staphylococcus isolados de leite de cabras do estado do Rio de Janeiro
}

\section{Enterotoxins production by Staphylococcus isolated from milk of goats from Rio de Janeiro state}

\author{
Francimara de Araújo Mariano, ${ }^{*}$ Marcio Manhães Folly, ${ }^{1 *}$ Gina Nunes Teixeira, ${ }^{*}$ Luiz Simeão do Carmo, ${ }^{* *}$ \\ Olney Vieira-da-Motta, ${ }^{*}$
}

\begin{abstract}
Resumo
Foram identificados Staphylococcus aureus e estafilococos coagulase-negativos produtores de enterotoxinas, a partir de amostras de leite de cabras. Coletaram-se 184 isolados estafilocócicos de 8 capris do estado do Rio de Janeiro. As amostras foram analisadas de acordo com técnicas microbiológicas de rotina e inoculadas em ágar estoque. A determinação das cepas enterotoxigênicas utilizou o método de Optimum Sensibility Plate (OSP). As cepas produtoras de enterotoxinas foram identificadas por método automatizado MiniApi pelo uso de galerias ID32Staph. Do total de amostras, 36 isolados (19,6\%) foram produtoras de enterotoxinas (A, B, C, D). As espécies identificadas foram: S. aureus $14(38,8 \%)$, S. capitis 1 (2,8\%), S. cohnii $2(5,4 \%)$, S. chromogenes 1 (2,8\%), S. lugdunensis $5(13,7 \%)$, S. haemolyticus $1(2,8 \%)$, S. simulans $5(13,7 \%)$, S. caprae $1(2,8 \%)$, S. warneri 1 (2,8\%) e S. epidermidis 5 (13,7\%). A toxina de maior ocorrência isolada foi SEA (27,8\%), seguido de SEC (19,4\%), SED $(11,1 \%)$ e SEB (8,3\%). As cepas produziram mais de uma toxina associada, SEBC $(16,7)$, SECD $(11,1 \%)$ e SEBCD $(5,6 \%)$. Os autores enfatizam a primeira ocorrência de $S$. caprae enterotoxigênica. Concluiu-se que bactérias coagulase-negativas produtoras de enterotoxinas constituem um risco iminente quando presentes no leite de cabras comercializado para consumo humano.
\end{abstract}

Palavras-chave: Staphylococcus, cabras, mastite, leite, enterotoxina.

\begin{abstract}
Strains of Staphylococcus aureus and coagulae-negative staphylococci producing enterotoxins of goat milk provenience were isolated. A total of 184 staphylococci strains coming from 8 breedings of the State of Rio de Janeiro were collected and analyzed in agreement with standardized microbiological routine techniques and inoculated onto agar stock. To determine SEs production the Optimum Sensibility Plate (OSP) method was used. Of that total, 36 strains $(19,6 \%)$ were enterotoxins secretors (A, B, C, D). The strains producing of enterotoxins were identified by the automated MiniAPI method in galleries ID32Staph: $S$. aureus 14 $(38,8 \%)$, S. capitis $1(2,8 \%)$, S. cohnii $2(5,4 \%)$, S. chromogenes $1(2,8 \%)$, S. lugdunensis $5(13,7 \%)$, S. haemolyticus $1(2,8 \%)$, S. simulans $5(13,7 \%), S$. caprae $1(2,8 \%)$, S. warneri $1(2,8 \%)$ e S. epidermidis $5(13,7 \%)$. Among the SEs detected, the most prevalent solely detected sorotype was SEA $(27,8 \%)$, followed by SEC $(19,4 \%)$, SED $(11,1 \%)$ and SEB $(8,3 \%)$. The associated SEs were SEBC (16,7), SECD (11.1\%) and SEBCD (5,6\%). The authors emphasize this is the first case of $S$. caprae enterotoxigenic. To conclude, the coagulase-negative staphylococci strains producing enterotoxins constitute a health risk when detected in goats milk commercialized for human consumption.
\end{abstract}

Keywords: Staphylococcus, goat, mastitis, milk; enterotoxin.

\section{Introdução}

O leite de cabra ocupa lugar de destaque devido ao seu alto valor nutritivo quando comparado ao leite de vaca devido a sua alta digestibilidade. Sendo, conseqüentemente, indicado para a alimentação de crianças e idosos impedidos de consumir leite de vaca, devido à intolerância à lactose (Egito e Pinheiro, 1989).

Mastite é o processo inflamatório da glândula mamária, que pode ser aguda ou crônica, onera a produção leiteira pela diminuição da produção de leite, custo com mão-de-obra, honorários profissionais, medicamentos, morte ou descarte precoce dos animais. Em laticínios ocorre queda na qualidade do produto final e diminuição no rendimento industrial para fabricação dos seus derivados, pela composição do leite alterado. Em sua etiologia, o gênero Staphylococcus figura entre os mais freqüentes (Langoni et al., 1998).

A intoxicação alimentar estafilocócica é uma doença caracterizada por episódios de gastroenterite aguda de evolução rápida e curta duração, não sendo raros os casos

*Laboratório de Sanidade Animal/CCTA/Universidade Estadual do Norte Fluminense. Av Alberto Lamego, 2000, Horto, Campos dos Goytacazes, Rio de Janeiro CEP 28013-602.

**ICB, UFMG, BH, Minas Gerais CEP 30161-970. 
de óbitos em neonatos e idosos (Bergdoll, 1990; Su e Lee Wong, 1997). É causada pelas enterotoxinas, também conhecidas por superantígenos (Frieman et al., 1993), pertencentes à família das toxinas pirogênicas que consistem em exoproteínas com baixo peso molecular e agrupadas de acordo com sua atividade biológica e propriedades físicoquímicas (Marrack e Kapler, 1990; Monday e Bohach, 1999). As enterotoxinas compreendem uma família de múltiplos sorotipos (Sea-See Seg-Sej) (Balaban e Rasooly, 2000; Dinges et al., 2000), e estudos complementados com as recentemente descobertas SEK, SEL, SEM, SEM, SEO, SEP, SEQ, SER e SEU (Jarraud et al., 2001; Letertre et al., 2003; Omoe et al., 2004 e 2005). Cada sorotipo foi designado de acordo com uma nomenclatura padrão na qual a enterotoxina descoberta é nomeada com uma letra na ordem de seu descobrimento (Monday e Bohach, 1999; Balaban e Rasooly, 2001). A enterotoxina $A$ (SEA) é responsável pela maioria dos casos de intoxicações em humanos, com doses tão baixas quanto $100 \mathrm{ng}$ da proteína, capaz de promover surtos alimentares (Evenson, et al., 1988) e uma concentração equivalente a $1 \mathrm{pg}$ por mililitro de SEA desencadeia a proliferação de linfócitos de ratos e humanos in vitro (Rasooly et al., 1997). Devido a suas características superantigênicas, as enterotoxinas promovem a linfoproliferação nos cultivos de células mononucleares periféricas do sangue (PBMC) de forma acentuada e não específica (Marrack e Kapler, 1990). A homologia, acima de $80 \%$, entre a enterotoxina $A$ (SEA) e a enterotoxina $E$ (SEE) foi demonstrada por semelhança entre as duas seqüências, o que permite que o anti-soro anti-SEA também detecte cepas SEE positivas (Lee et al., 1978).

Acreditava-se que $S$. aureus era a única espécie conhecida como produtora de enterotoxinas. Uma importante característica que diferencia o $S$. aureus das outras espécies é a habilidade de produzir coagulase. Outras espécies nãoaureus coagulase positivas e negativas foram identificadas como enterotoxigênicas (Su e Lee Wong, 1997; Pereira et al., 2001). No entanto, quando leite de cabras na Itália foi examinado, as cepas estafilocócicas produtoras de enterotoxinas pertenciam apenas à espécie $S$. aureus, embora a maioria $(90 \%)$ dos isolados fossem coagulase-negativas (Foschino et al., 2002). Em rebanhos leiteiros do estado do Rio de Janeiro foram isolados $S$. aureus com clones predominantes, porém específicos de animais, como caprinos, bovinos, bublainos e ovinos (Aires-de-Sousa et al., 2007) e entre as cepas caprinas algumas foram caracterizadas como enterotoxigênicas (Silva, 2005).

Objetivou-se neste estudo isolar e identificar cepas de Staphylococcus sp e determinar os sorotipos de enterotoxinas produzidos pelas mesmas provenientes de cabras com mastite subclínica em rebanhos do Estado do Rio de Janeiro.

\section{Material e métodos}

Foram coletadas 184 amostras de leite em oito capris das regiões Norte e Serrana do estado do Rio de Janeiro, sendo cinco da região Serrana e três da região Norte, no período de março de 1999 a fevereiro de 2000.

Após higienização dos tetos por lavagem com água e sabão de coco e desinfecção com álcool $70^{\circ} \mathrm{C}$, os primeiros jatos de leite foram desprezados e 5,0 mL de leite foram coletados em tubos de ensaio estéreis, previamente identificados, acondicionados em caixa de isopor com gelo e transportados ao Laboratório de Sanidade Animal/CCTA/UENF onde foram incubados em estufa bacteriológica por 18 horas a $37^{\circ} \mathrm{C}$.

Após o período de incubação, foram usados os seguintes meios de cultura: Ágar Sangue, Chapmman, MacConckey e Sabouraud (Merck, Alemanha). Os cultivos foram incubados em estufa bacteriológica $\left(37^{\circ} \mathrm{C}\right)$ com observação do crescimento bacteriano após 24 e 48 horas após incubação. Decorrido este tempo, procedeu-se à análise morfotintorial das colônias pelo Gram. Em seguida, realizaram-se as provas bioquímicas específicas, das colônias identificadas como Gram-positivas e cocos com aspecto de cachos. Selecionaram-se então as bactérias que apresentaram os seguintes resultados nas provas bioquímicas: Catalase positiva, VogesProskauer positivo, fermentação do manitol em anaerobiose positivo, produção de oxidase negativa. Os resultados para a produção de coagulase em tubo contendo plasma de coelho EDTA (Dico, EUA) foram todos considerados, tanto os negativos quanto os positivos.

Extração de enterotoxinas pelo método de Membrana Sobre Agar - MSA (Robbins et al., 1974):

Vinte círculos, do diâmetro de uma placa de Petri (100X15 $\mathrm{mm}$ ), foram cortados de membrana de diálise (cut off $14 \mathrm{KDa}$, Sigma, EUA) e dispostos alternadamente com papel-filtro em uma outra placa de Petri (140X20 mm), embebidos em água destilada e autoclavados por 20 minutos a $121^{\circ} \mathrm{C}$. As membranas estéreis foram assepticamente transferidas para placas de Petri contendo $20 \mathrm{~mL}$ de ágar BHA.

As amostras a serem testadas foram inoculadas em $\mathrm{BHI}$ e após 24 horas a $37^{\circ} \mathrm{C}, 100 \mathrm{~mL}$ do cultivo foram espalhados sobre as membranas dispostas sobre o BHA com o auxílio da alça de Drigalski. As placas foram incubadas por 24 horas à $37^{\circ} \mathrm{C}$. Após este período, a massa celular da superfície da membrana foi lavada com 2,0 mL de solução tampão PBS $\left(\mathrm{NaHPO}_{4}\right.$ a 0,01 M, ph 7,4) e a suspensão obtida foi colocada em tubos de polipropileno e centrifugada a 5000 rpm por 20 minutos em temperatura ambiente. Em seguida, o sobrenadante foi transferido para um segundo tubo de polipropileno para pesquisa de enterotoxinas através do método de Optimum Sensibility Plate (OSP), por imunodifusão (Robbins et al., 1974). Para tal, placas de polipropileno de $50 \mathrm{~mm} \times 12 \mathrm{~mm}$ (Falcon, EUA) foram utilizadas contendo 3,0 $\mathrm{mL}$ por placa de ágar Noble a 1,2\% (Difco, EUA) preparado em PBS adicionado de solução de Timerosal (1:1000) na quantidade de $100 \mathrm{~mL}$ para cada $100 \mathrm{~mL}$ de meio. Após a solidificação do meio a $4^{\circ} \mathrm{C}$ por 15 minutos, poços foram perfurados com auxílio de um molde de acrílico (padrão FRIFood Research Institute). Os botões de ágar foram removidos por sucção com auxílio de pipeta Pasteur e bomba a vácuo. As placas foram identificadas com as letras: A, B, C, D para cada anti-soro e respectivas toxinas-padrão utilizados nos testes (obtidos na FUNED, MG). No poço central contendo anti-soro $(50 \mathrm{~mL})$ e cada enterotoxina controle $(25 \mathrm{~mL})$ colocadas nos dois poços menores, e as amostras testadas $(50 \mathrm{~mL})$ nos quatro poços maiores e amostras-padrão (FRI100-SEA, FRI361-SEC, FRIS-6 SEB e FRI1151m-SED) produtoras de cada sorotipo testado. Em seguida, as placas fechadas foram colocadas em repouso em câmara úmida, a $37^{\circ} \mathrm{C}$. A leitura foi registrada por até 48horas de incubação. Este teste possui limite de detecção de $0,5 \mathrm{mg} / \mathrm{ml}$ de amostra (Robbins et al., 1974). 
A identificação dos 36 isolados enterotoxigênicos foi realizada com uso de galerias do Kit ID32 STAPH (bioMérieux, França). Cada amostra bacteriana teve a concentração ajustada correspondente a 0,5 da escala MacFarland medido por fotometria em leitor equipado para leitura de densidade óptica a 550nm (Densimat, bioMérieux, França) de acordo com as especificações do fabricante. A interpretação dos resultados foi feita pelo uso do software MiniApi (bioMérieux, Itália).

\section{Resultados e discussão}

O trabalho identificou Staphylococcus spp. produtores de enterotoxinas. A Tabela 1 mostra os resultados obtidos nos oito capris demonstrando que a grande maioria das bactérias isoladas e testadas para produção de enterotoxinas, 162 $(88,0 \%)$ foram coagulase-negativas e 22 (12\%) foram coagulase-positivas. Concordando com o que foi demonstrado em 1990, num estudo em quatro rebanhos da raça Saanen que obteve como resultado espécies bacterianas isoladas predominantes sendo os Staphylococcus $s p$ coagulasenegativa, num total de 896 amostras analisadas, 684 fizeram parte deste grupo. As infecções devido a este tipo de bactéria variaram de $9,2 \%$ a $22,6 \%$ das metades mamárias nos quatro rebanhos estudados (Ryan e Greenwood, 1990).

Tabela 1: Distribuição das 186 amostras de Staphylococcus spp coagulase-positiva e negativa testadas para produção de enterotoxinas nos rebanhos da região Norte e Serrana do estado do Rio de Janeiro de março de 1999 a fevereiro de 2000.

\begin{tabular}{|c|c|c|}
\hline \multicolumn{3}{|c|}{ TESTE DE COAGULASE } \\
\hline & POSITIVO \%(n) & NEGATIVO \%(n) \\
\hline CAPRIS & & \\
\hline A & $50(11)$ & $48,1(78)$ \\
\hline B & $18,2(4)$ & $22,2(36)$ \\
\hline C & $0(0)$ & $1,2(2)$ \\
\hline D & $0(0)$ & $5,6(9)$ \\
\hline$E$ & $27,3(6)$ & $18,6(30)$ \\
\hline $\mathrm{F}$ & $0(0)$ & $3,7(6)$ \\
\hline G & $0(0)$ & $0,6(1)$ \\
\hline $\mathrm{H}$ & $4,5(1)$ & $0(0)$ \\
\hline TOTAL & $100(22)$ & $100(162)$ \\
\hline
\end{tabular}

Dentro do grupo das 184 bactérias testadas pelo método OSP, $36(19,56 \%)$ foram positivas, produzindo um ou mais sorotipo de enterotoxinas. As 148 cepas restantes foram negativas para produção de enterotoxinas. Na Tabela 2 está especificado o total de amostras produtoras e não-produtoras de enterotoxinas, bem como o resultado para prova da coagulase.

Conforme os dados da Tabela 2, a grande maioria das cepas enterotoxigênicas $(61,1 \%)$ foi coagulase-negativa, o que vem a corroborar com Su e Lee Wong (1997) que afirmam que a capacidade para produção de coagulase não pode ser considerada como indicativo de enterotoxigenicidade.

Uma alta relação entre produção de coagulase e de enterotoxinas tem sido relatada (Bedidi-Madini et al, 1998; VERNOZY et al., 1996). No entanto, esta afirmação não é mais absoluta, considerando-se os trabalhos que identificaram diversas cepas de Staphylococcus $s p$ coagulase-negativas produtoras de enterotoxinas (Vernozy et al., 1996; Su e Lee
Wong, 1997) e que também pode ser observado nos resultados deste trabalho.

Ainda na Tabela 2 está demonstrado o resultado para o teste OSP bem como a produção dos diversos sorotipos das enterotoxinas estafilocócicas produzidos isoladamente ou em conjunto pelos 184 isolados de Staphylococcus sp.

Tabela 2: Determinação de enterotoxinas pelo método de OSP de 36 cepas de Staphylococcus sp. provenientes de leite de cabras da região Norte e Serrana do estado do Rio de Janeiro no período de março a fevereiro de 2000.

\begin{tabular}{cccc}
\hline SOROTIPOS & \multicolumn{2}{c}{ COAGULASE } & $\begin{array}{c}\text { TOTAL DE } \\
\text { CEPAS }\end{array}$ \\
\hline \multirow{4}{c}{ Negativa \%(n) } & Positiva\%(n) \\
A & $40,9(9)$ & $7,1(1)$ & $27,8(10)$ \\
B & $13,6(3)$ & 0 & $8,3(3)$ \\
C & $9,1(2)$ & $35.8(5)$ & $19,4(7)$ \\
D & $13,6(3)$ & $7.1(1)$ & $11.1(4)$ \\
B e C & $18,2(4)$ & $14,3(2)$ & $16.7(6)$ \\
C e D & $4,6(1)$ & $21,4(3)$ & $11.1(4)$ \\
B, C e D & 0 & $14,3(2)$ & $5.6(2)$ \\
TOTAL & $100(22)$ & $100(14)$ & $100(36)$ \\
\hline
\end{tabular}

Schocken-Iturrino et al. (1986), analisando amostras de leite de vacas em nove propriedades no município de Jaboticabal/ $\mathrm{SP}$, verificaram a capacidade produtora de enterotoxinas dos tipos A, B, C, D e E, efetuadas nos 19 isolados de $S$. aureus e evidenciaram a presença de três amostras $(15,79 \%)$ enterotoxigênicas, as quais foram produtoras de SEC. Os estudos realizados com leite de búfalas em seis rebanhos do estado do Rio de Janeiro demonstraram pela primeira vez a presença de $S$. aureus produtores de enterotoxina $C$ (SEC) e a Toxina da Síndrome do Choque Tóxico-1 (TSST-1), avaliados por imunodifusão (Ferreira et al., 2007). O mesmo grupo de pesquisadores discutiu sobre a possível transferência de cepas estafilocócicas de bovinos e caprinos, além da circulação de cepas enterotoxigênicas, entre os bubalinos foi considerada devido ao manejo e criação mista entre espécies de ruminantes em algumas propriedades pesquisadas (Bonna et al., 2004). Estes isolados de $S$. aureus presentes em leite de rebanhos de ruminantes, entre eles caprinos, estudados no estado do Rio de Janeiro foram analisados molecularmente pela técnica de PFGE e observouse a prevalência de um clone majoritário (Aires-de-Sousa et al., 2007). As cepas de Staphylococcus spp. isoladas neste trabalho estariam secretando SEA e SEE isoladamente ou ambas, em proporção ainda não detectada, pois a homologia entre estes sorotipos foi observada a partir de análises cromatográficas e o reconhecimento de anticorpos comuns às duas moléculas (Lee et al., 1978). Neste trabalho, foram identificadas pelo aparelho MiniApi 36 bactérias enterotoxigênicas do gênero Staphylococcus. Da espécie $S$. aureus, 14 foram produtoras de enterotoxinas seja de um sorotipo ou de mais de um simultaneamente. E dentro do grupo das coagulase negativas 22 foram enterotoxigênicas, como mostrado na Tabela 3. Ferreira et al. (2007), ao analisarem leite de búfalas criadas em propriedades de aptidão mista (búfalos, caprinos e bovinos) no estado do Rio de Janeiro, também isolaram $S$. aureus secretores de mais de um sorotipo, simultaneamente. 
Tabela 3: Identificação automatizada de 36 cepas de Staphylococcus sp enterotoxigênicas provenientes de leite de cabras com mastite subclínica das regiões Norte e Serrana do estado do Rio de Janeiro durante o período de março a fevereiro de 2000.

\begin{tabular}{lcccccccc}
\hline & & \multicolumn{7}{c}{ SOROTIPOS } \\
\hline Espécies & $\mathrm{n}$ & SEA & SEB & SEC & SED & SEBC & SECD & SEBCD \\
\hline S. aureus & 14 & 1 & 0 & 5 & 1 & 2 & 3 & 2 \\
S.cohnii & 2 & 1 & 0 & 1 & 0 & 0 & 0 & 0 \\
$\quad$ S. capitis & 1 & 0 & 1 & 0 & 0 & 0 & 0 & 0 \\
S. chromogenes & 1 & 0 & 0 & 0 & 0 & 1 & 0 & 0 \\
S. lugdunensis & 5 & 1 & 0 & 0 & 2 & 2 & 0 & 0 \\
$\quad$ S. simulans & 5 & 2 & 1 & 0 & 1 & 1 & 0 & 0 \\
S. epidermidis & 5 & 3 & 1 & 0 & 0 & 0 & 1 & 0 \\
S. caprae & 1 & 1 & 0 & 0 & 0 & 0 & 0 & 0 \\
S. warneri & 1 & 0 & 0 & 1 & 0 & 0 & 0 & 0 \\
S. haemolyticus & 1 & 1 & 0 & 0 & 0 & 0 & 0 & 0 \\
$\quad$ TOTAL & 36 & 10 & 3 & 7 & 4 & 6 & 4 & 2 \\
$\quad \%$ & & 27,8 & 8,3 & 19,4 & 11,1 & 16,7 & 11,1 & 5,6 \\
\hline
\end{tabular}

SE: Enterotoxina Estafilocóccica A, B, C, D.

A maioria das cepas de Staphylococcus spp. enterotoxigênicas produziu SEC isolada ou em combinação com outro sorotipo. Valle et al., (1990) encontraram 22\% das amostras estafilocócicas coagulase-negativas testadas como sendo produtoras de enterotoxinas e as espécies identificadas foram: $S$. chromogenes produtora somente de SEC e $S$. caprae, $S$. xylosus, S. sciuri, S. saprophyticus, S. lentus, S. warneri, S. epidermidis e $S$. haemolyticus. Das espécies citadas, algumas foram identificadas no presente trabalho, com exceção de $S$. sciuri e $S$. lentus.

$S$. aureus SEA-positivo é um dos mais prevalentes em surtos de intoxicação alimentar em humanos (Archer e Young, 1988). Conforme demonstrado na Tabela 3, SEA foi o mais produzido isoladamente $(27,8 \%)$ e não simultaneamente com outro sorotipo. Entre as espécies coagulase-positivas foram isoladas 14 cepas de Staphylococcus aureus enterotoxigê-nicas e destas, cinco foram produtoras de SEC $(35,8 \%)$, uma de SEA $(7,1 \%)$ e SED $(7,1 \%)$, duas de SEBC $(14,3 \%)$ e SEBCD $(14,3 \%)$ e três de SECD (21,4\%). A ocorrência de toxinfecção alimentar em crianças devido ao consumo de leite de cabra com mastite por $S$. aureus foi relatada em Israel (Gross et al., 1988). Em análises microbiológicas de leite de ovelhas, Orden et al., (1992) obtiveram apenas cepas de $S$. aureus secretoras de SEC em combinação com outro superantígeno estafilocócico, a Toxina do Choque Tóxico-1 (TSST-1) em $74 \%$ das 160 amostras estafilocócicas estudadas e nenhuma cepa coagulase-negativa mostrou caráter toxigênico. Silva et al. (2005) observaram diferentes isolados estafilocócicos enterotoxigênicos em

Tabela 4: Produção de diferentes sorotipos de enterotoxinas por cepas estafilocócicas isoladas de leite de animais portadores de mastite, em diferentes locais.

\begin{tabular}{ccccc}
\hline & \multicolumn{3}{c}{ BACTÉRIA/SOROTIPOS } \\
AUTOR/REFERÊNCIA & $\begin{array}{c}\text { Staphylococcus } \\
\text { aureus }\end{array}$ & $\begin{array}{c}\text { Staphylococcus } \\
\text { coagulase negativa }\end{array}$ & $\begin{array}{c}\text { FONTE/ESPÉCIE } \\
\text { ANIMAL }\end{array}$ \\
FREITAS et al., 1990 & A & - & Vacas \\
NADER FILHO et al.,1988 & B & - & Vacas \\
MOTTA, 2001 & A, B, C, D & - & Vacas \\
GROSS et al., 1988 & B & - & Cabras \\
VALLE et al., 1990 & & A, B, C & Cabras \\
VERNOZY-ROZAND et al., 1996 & A & - & Cabras \\
BONNA et al., 2004 & C & - & Búfalas \\
FERREIRA et al, 2007 & C, B, C, D & A, B, C, D & Ovelfalas \\
SILVA et al. 2005 & C cabras & Ovelhas \\
ORDEN et al., 1992 & A, C, D & A, B, C & Cabras \\
Este trabalho & & &
\end{tabular}

leite reagentes ao $\mathrm{CMT}$, com apenas uma cepa SEB positiva (Nader Filho et al., 1988). Em outros três municípios do estado de São Paulo, foram pesquisadas 1662 vacas leiteiras de 20 propriedades e as enterotoxinas estavam presentes em menos de $5 \%$ dos isolados de $S$. aureus, com SEB, SEC e SED identificadas por aglutinação em látex (Pinheiro-de-Sá et al., 2004). No estado do Rio de Janeiro, foram analisadas 93 isolados de $S$. aureus de leite de vacas e apenas uma foi produtora de SEA, considerada a de maior ocorrência nas intoxicações alimentares humanas (Freitas e Magalhães, 1990). Os estudos realizados com 61 isolados de $S$. aureus presentes em leite de vacas de 16 municípios da Região Norte/Noroeste do Estado do Rio de Janeiro demonstraram que 16 (24\%) delas produziram enterotoxinas e houve a predominância de SED (seis isolados), seguido SEA (quatro isolados) e SEC (um isolado), e isolados com dupla produção (três SEA+SED, uma SEB+SED e uma SEC+SED) examinadas através de imunodifusão, immunobloting e ELISA (Motta, 2001).

$\mathrm{Na}$ Tabela 3 também foi demonstrado o $S$. caprae produtor de enterotoxina $A$, fato até então não citado na literatura. Foschino et al. (2002), ao examinarem leite caprino na região de Bérgamo, Itália, identificaram 26 isolados de $S$. aureus dos quais $23 \%$ foram enterotoxigênicos com prevalência para SEC e 54 cepas de Staphylococcus coagulase-negativas e nenhuma produtora de enterotoxinas. A Tabela 4 ilustra alguns trabalhos com cepas estafilocócicas secretoras de enterotoxinas, isoladas de países, fontes/espécies diferentes. leite de cabras e ovelhas da região norte

do estado do Rio de Janeiro. De rebanhos bubalinos leiteiros, no estado do Rio de Janeiro, foram detectados isolados de $S$. aureus secretores de SEC e TSST-1 associadas (Ferreira et al., 2007). Em Ribeirão Preto-SP, em 17 propriedades com 497 vacas, avaliou-se a capacidade de produção de enterotoxinas de 74 isolados de $S$. aureus em amostras de 
e sua conseqüente resistência ao tratamento de pasteurização do leite, o produto comercializado pode representar um risco à saúde pública. $O$ sorotipo $A$ (SEA) foi o de maior ocorrência entre as amostras estudadas e é citado na literatura como o mais incriminado nos quadros de intoxicação alimentar.

\section{Agradecimentos}

À Profa. Dra. Helena R. Lopes, da Universidade Federal Fluminense, por ceder as cepas-padrão de S. aureus. A FENORTE, pela bolsa concedida a Francimara de Araújo Mariano, e à FAPERJ pelo apoio financeiro a Olney Vieira-da-Motta, processo E-26/ $170.509 / 2004$.

\section{Referências}

AIRES-DE-SOUSA, M.; PARENTE, C.E.S.R.; VIEIRA-DA-MOTTA, O. et al. Characterization of Staphylococcus aureus isolated from buffalo, bovine, ovine, and caprine milk samples from Rio de Janeiro State, Brazil. Appl. Environ. Microbiol., v. 73, p. 3845-3849, 2007.

ARCHER, D.L.; YOUNG, F.E. Contemporary issues: disease with a food vector. Clin. Microbiol. Rev., v. 1, p. 377-398, 1988.

BALABAN, N.; RASOOLY, A. Staphylococcal enterotoxins. Intl. J. Food Microbiol., v. 61, p.1-10, 2000.

BEDIDI-MADANI, N.; GREENLAND, T.; RICHARD, Y. Exoprotein and slime production by coagulase-negative staphylococci isolated from goats' milk. Vet. Microbiol., v. 59, p. 139-145, 1998.

BERGDOLL, M. S. Analytical methods for Staphylococcus aureus. Intl. J. Food Microbiol., v. 10, p. 91-99, 1990.

BONNA, I. C. F.; FERREIRA, G. S.; CARMO, L. S. et al. Estudo da microbiota da glândula mamária de búfalas, com ênfase para Staphylococcus aureus produtores de toxinas. Rev. Univ. Rural-Sér. Ciên. Vida, v. 24, p. 485-486, 2004.

CASMAN, E. P.; BEGDOLL, M. S.; ROBINSON, J. Designation of staphylococcal enterotoxins. J. Bacteriol., v. 85, p. 715-716, 1962.

DINGES, M. M.; ORWIN, P. M.; SCHLIEVERT, P. M. Exotoxins of Staphylococcus aureus. Clin. Microbiol. Rev., v. 13, p. 16-34, 2000.

EGITO, A. S.; PINHEIRO, R. R. Produção higiênica do leite de cabras. Sobral-CE: EMBRAPA - CNPC, Comunicado Técnico, n. 20, 1989.

EVENSON, M. L.; HINDS, M. W.; BERNSTEIN, R. S. et al. Estimation of human dose of staphylococcal enterotoxin A from a large outbreak of staphylococcal food poisoning involving chocolate milk. Intl. J. Food Microbiol., v. 7, p. 311-316, 1988.

FERREIRA, G. S.; BONNA, I. C. F.; CARMO, L. S. et al. Desenvolvimento de anticorpos IgY contra superantígenos de Staphylococcus aureus isolados de leite de búfalas de rebanhos do Estado do Rio de Janeiro. Rev. Univ. Rural-Sér. Ciên. Vida, v. 27, p. 598-600, 2007.

FOSCHINO, R.; INVERNIZZI, A.; BARUCCO, R. et al. Microbial composition, including the incidence of pathogens, of goat milk from the Bergamo region of Italy during a lactation year. J. Dairy Res, v. 69, p. 213-225, 2002.

FREITAS, M. A. Q. de, MAGALHÃES, H. Enterotoxigenicidade de Staphylococcus aureus, isolados de vacas com mastite. Rev. Microbiol., v. 21, p. 315-319, 1990.

FRIEMAN, S. M.; TUMANG, J. R.; CROW, M. K. Microbial superantigens as etiopathogenic agents in autoimmunity. Rheum. Dis. Clin North Am., v. 19, p. 207-222, 1993.

GROSS, E. M.;WEIZMAN, Z;; PICARD, E. et al. Milkborne gastroenteritis due to Staphylococcus aureus enterotoxin $B$ from a goat with mastitis. Am. J. Trop. Med. Hyg., v. 39, p. 103-104, 1988.

JARRAUD, S.; PEYRAT, M. A.; LIM, A. et al. egc, a high prevalent operon of enterotoxin gene, forms a putative nursery of superantigens in Staphylococcus aureus. J. Immun., v. 166, p. 669-677, 2001.

LANGONI, H.; SILVA, A. V. da; CABRAL, K. G. et al. Aspectos etiológicos na mastite bovina: flora bacteriana aeróbica. Rev. Bras. Med. Vet., v. 20, p. 204-208, 1998.
LEE, A. C.; ROBBINS, R. N.;BERGDOLL, M. S. Isolation of specific and common antibodies to staphylococcal enterotoxins $A$ and $E$ by affinity chromatography. Infect. Immun., v. 21, p. 387-391, 1978.

LETERTRE, C.; PERELLE, S.; DILASSER, F. et al. Identification of a new putative enterotoxin SEU encoded by the egc cluster of Staphylococcus aureus. J. Appl. Microbiol., v. 95, p. 38-43, 2003.

MARRACK, P.; KAPPLER, J. The staphylococcal enterotoxins and their relatives. Science, v. 248, p. 705-711, 1990.

MONDAY, S. R.; BOHACH, G. A. Properties of Staphylococcus aureus enterotoxins and toxic shock syndrome toxin-1. In: ALOUF, J. E., FREER, J. H. (Ed.) The comprehensive Sourcebook of Bacterial Protein toxins. EUA: San Diego, 1999. p. 589-610.

MOTTA, O. V. O "Quorum sensing" dos mecanismos de expressão de enterotoxinas de Staphylococcus aureus como foco de terapias alternativas para mastite bovina. 2001. 160 f. Dissertação (Doutorado em Biociências e Biotecnologia) - Centro de Biociências e Biotecnologia, Universidade Estadual do Norte Fluminense Darcy Ribeiro, Campos dos Goytacazes.

NADERFILHO, A.;ROSSI Jr,O. D.; SCHOCKEN-ITURRINO, R. P. Pesquisa de Staphylococcus aureus, enterotoxigênicos em vacas com mastite subclínica. Rev.Bras. Microbiol., v. 19, p. 369-373, 1988.

OMOE, K.; IMANISHI, K.; HU, D. L. et al. Biological properties of staphylococcal enterotoxin-like toxin type R. Infect. Immun., v. 72, p. 3664-3667, 2004.

OMOE, K.; IMANISHI, K.; HU, D. L. et al. Characterization of novel staphylococcal enterotoxin-like toxin type P. Infect. Immun., v. 73, p. 5540-5546, 2005.

ORDEN, J. A.; CID, D.; BLANCO, M. E. et al. Enterotoxin and toxic shock syndrome toxin-one production by staphylococci isolated from mastitis in sheep. APMIS, v. 100, p.132-134, 1992.

PEREIRA, M. L., CARMO, L. S., PEREIRA, J. L. Comportamento de estafilococos coagulase negativos pauciprodutores de enterotoxinas, em alimentos experimentalmente inoculados. Ciênc. Tecnol. Aliment., v. 21, p. 171-175, 2001.

PINHEIRO-DE-SÁ, M. E.; CUNHA, M. L. R. S.; ELIAS, A. O. et al. Importância do Staphylococcus aureus nas mastites subclínicas: pesquisa de enterotoxinas e toxina do choque tóxico, e a relação com a contagem de células somáticas. Braz. J. Anim. Sci., v. 41, p. 320326, 2004.

RASOOLY, L.; ROSE, N. R.; SHAH, D. B. et. al. In vitro assay of Staphylococcus aureus enterotoxin A activity in food. Appl. Envir. Microbiol., v. 63, p. 2361-2365, 1997.

ROBBINS, R.; GOULD, S.; BERGDOLL, M. S. Detecting the enterotoxigenicity of Staphylococcus aureus strains. Appl. Microbiol., v. 28, p. 946-950, 1974.

RYAN, D. P.; GREENWOOD, P. L. Prevalence of udder bacteria in milk samples from four dairy goat herds. Aust. Vet. J., v. 67, p. 362-363, 1990.

SCHOCKEN-ITURRINO, R. P.; NADER FILHO, A.; FURLANETTO, S. M. P. et al. Pesquisa de Staphylococcus aureus enterotoxigênicos em amostras de leite de vacas mastíticas. Ars Vet., v. 2, p. 69-74, 1986. 
SILVA, D. A.; FERREIRA, G. S.; CARMO, L. S. et al. Produção e Detecção de TSST-1 e enterotoxinas em cepas estafilocócicas isoladas de leite de ovelhas e cabras do Norte Fluminense pelos métodos de imunodifusão e PCR. In: XXIII Congresso Brasileiro de Microbiologia, 2005, Santos. ANAIS XXIII CONGRESSO BRASILEIRO DE MICROBIOLOGIA, Santos-SP:2005. (Resumo).
SU, Y. C.; LEE WONG, A C. Current perspectives on detection of staphylococcal enterotoxins. J. Food Prot., v. 60, p. 195-202, 1997.

VALLE, J.; GOMEZ-LUCIA, E.; PIRIZ, S. et al. Enterotoxin production by staphylococci isolated from healthy goats. Appl. Envir. Microbiol. v. 56, p. 1323-1326, 1990.

VERNOZY-ROZAND, C.; MAZUY, C; PREVOST, G. et al. Enterotoxin production by coagulase-negative staphylococci isolated from goats'milk and cheese. Intl. J. Food Microbiol., v. 30, p. 271-280, 1996. 